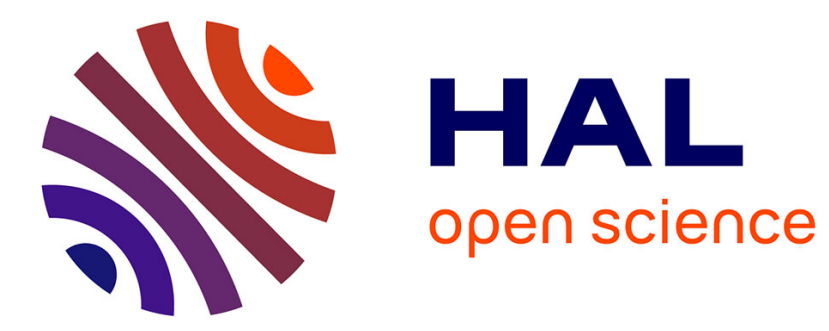

\title{
2D Robust Magnetic Resonance Navigation of a Ferromagnetic Microrobot using Pareto Optimality
}

\author{
David Folio, Antoine Ferreira
}

\section{To cite this version:}

David Folio, Antoine Ferreira. 2D Robust Magnetic Resonance Navigation of a Ferromagnetic Microrobot using Pareto Optimality. IEEE Transactions on Robotics, 2017, 99, pp.1-11. 10.1109/TRO.2016.2638446 . hal-01446482

\section{HAL Id: hal-01446482 \\ https://hal.science/hal-01446482}

Submitted on 26 Jan 2017

HAL is a multi-disciplinary open access archive for the deposit and dissemination of scientific research documents, whether they are published or not. The documents may come from teaching and research institutions in France or abroad, or from public or private research centers.
L'archive ouverte pluridisciplinaire HAL, est destinée au dépôt et à la diffusion de documents scientifiques de niveau recherche, publiés ou non, émanant des établissements d'enseignement et de recherche français ou étrangers, des laboratoires publics ou privés. 


\title{
2D Robust Magnetic Resonance Navigation of a Ferromagnetic Microrobot using Pareto Optimality
}

\author{
David Folio and Antoine Ferreira
}

\begin{abstract}
This paper introduces a two-dimensional autonomous navigation strategy of a $750 \mu \mathrm{m}$ steel microrobot along a complex fluidic vascular network inside the bore of a clinical $3.0 \mathrm{~T}$ magnetic resonance imaging (MRI) scanner. To ensure successful magnetic resonance navigation (MRN) of a microrobot along consecutive channels, the design of autonoumous navigation strategy is needed taking into account the major MRI technological constraints and physiological perturbations, e.g. non-negligible pulsatile flow, limitations on the magnetic gradient amplitude, MRI overheating, susceptibility artifacts uncertainties, and so on. An optimal navigation planning framework based on Pareto optimality is proposed in order to deal with this multiple-objective problem. Based on these optimal conditions, a dedicated control architecture has been implemented in an interventional medical platform for real-time propulsion, control and imaging experiments. The reported experiments suggest that the likelihood of controlling autonomously untethered $750 \mu \mathrm{m}$ magnetic microrobots is rendered possible in a complex twodimensional centimeter-sized vascular phantom. The magnetic microrobot traveled intricate paths at a mean velocity of about $4 \mathrm{~mm} \mathrm{~s}^{-1}$ with average tracking errors below $800 \mu \mathrm{m}$ with limited magnetic gradients $\pm 15 \mathrm{mT} \mathrm{m}^{-1}$ compatible with clinical MRI scanners. The experiments demonstrate that it is effectively possible to autonomously guide a magnetic microrobot using a conventional MRI scanner with only a software upgrade.
\end{abstract}

Index Terms-Microrobotics, Magnetic Resonance Imaging, Magnetic Resonance Navigation, Multi-Ojective Planning.

\section{INTRODUCTION}

$\mathbf{M}$ ICROROBOTICS is a promising solution for biomedical application innovation, including targeted therapies, diagnosis, drug delivery, minimally invasive surgery, or cells transportation [1], [2]. To control microrobotic devices within the human body, magnetic actuation is till now the most advanced solution [2]. Currently, different concepts have been proposed using specific magnetic manipulation systems [3], [4] or upgraded clinical Magnetic Resonance Imaging (MRI) scanner [5]-[11]. The former usually provides up to 5 degrees of freedom with great steering accuracy. However, they have limited medical application as they provide low magnetic field and require an external vision system. In contrast, the second approach requires only an upgrade of current software and/or hardware capabilities of clinical MRI systems. Initially devoted to medical imaging, MRI scanners are nowadays investigated to become an assisting tool for navigation control in vascular networks. In cancer therapy, therapeutic agents are injected using a magnetic catheter to impose a starting point for the navigable path of the agents to be located within an artery. As shown in Fig. 1, to reach a tumor, therapeutic

D. Folio and A. Ferreira are with the INSA Centre Val de Loire, Université d'Orléans, PRISME EA 4229, Bourges, France. (e-mail: david.foliodinsa-cvl.fr; antoine.ferreiraeinsa-cvl.fr)

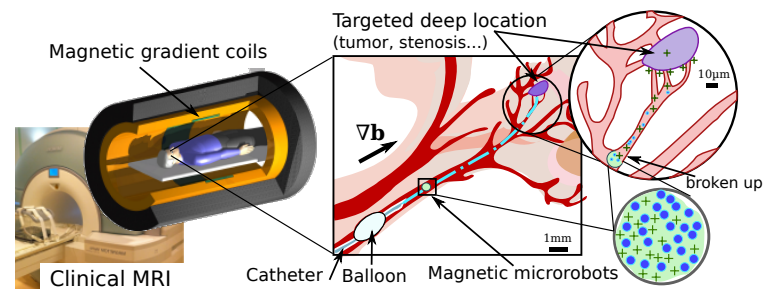

Fig. 1. Representation of the Magnetic Resonance Navigation (MRN): magnetic microrobots are released in artery from a catheter. By correctly applying magnetic gradients $\nabla \mathbf{b}$ from the MRI scanner, microrobots follow an optimal planned path. In tiny vessels, microrobots are broken up and drugs are released to a targeted area.

microagents are transiting through arteries (cm-wide), narrower arterioles before reaching the tumoral regions accessible through the capillaries ( $\mu \mathrm{m}$-wide) forming the angiogenesis network. To achieve such a goal, robust and safe endovascular navigation presents challenging issues in terms of modeling and control [8].

Actually, the main research efforts are carried out in the design of robust and autonomous magnetic resonance navigation (MRN) strategies easily implementable in clinical MRI scanners. Basically, MRN can be performed using two fundamental approaches namely, Imaging Gradient Coil (IGC) or Steering Gradient Coil (SGC). The former consists of upgrading a clinical MRI scanner with the software only. A breakthrough in interventional MRI procedures demonstrated that real-time IGC systems can offer a well-suited environment for the imaging, tracking, and control of a magnetic microrobot, which was done with a pig [5] and a rabbit [9]. The sole MRI software upgrade does not imply any major modifications, and allows keeping it for conventional operations. In this context, the MRN relies on the use of gradient coils of the MRI for imaging and propulsion. By alternating these two sequences it has been demonstrated that it is possible to guide magnetic microrobots along a simple path using a clinical MRI scanner [6], [11]. These successful in vivo experiments pointed out critical challenges in terms of real-time imaging and tracking, together with optimal navigation strategy for future therapeutic applications [10]. Furthermore, the gradients used for the whole body imaging are relatively low (e.g. $<80 \mathrm{mT} \mathrm{m}^{-1}$ ) for conventional scanners. In contrast, the SGC implies integrating additional coils capable to generate higher gradients, typically more than $300 \mathrm{~m} \mathrm{~T} \mathrm{~m}^{-1}$ while improving the magnetic steering and imaging capacities. The key to this innovative system is the capability to concurrently use powerful insert coils with the body magnet's gradient coils. As illustration, the authors in [12] developed the highest strength insert imaging gradient 
coil that allows peak strength values of $600 \mathrm{mT} \mathrm{m}^{-1}$ with fast rise time of $5 \mathrm{~ms}$. Similarly, Martel et al. proposed to improve the MRI hardware by adding additional electromagnetic coils [9] delivering gradients of $300 \mathrm{~m} \mathrm{~T} \mathrm{~m}^{-1}$ allowing MR-imaging or MR-tracking in a time-multiplexed way. However, such supplementary gradient inserts lead to a reduction of the MRI bore, and usually limit the MRN application to human members or small animals. New versions of ultra-high gradients scanners for whole body MRN are actually developed. The possibility to perform MRN within a whole-body MRI has been recently demonstrated by modifying a Mangetom Skyra 3T magnet [13]. For these upgraded steering gradient coil setups, technological constraints related to cooling time of the steering gradient coils lead to limited duty cycles of the imaging-propulsion sequences that greatly affects the robustness and stability of the real-time control loop.

Our approach in this work is to improve the standard IGCbased MRN strategy in complex phantom vascular models using a standard clinical MRI scanner without hardware modifications. Although weaker driving gradient forces are generated, the propulsion of magnetic microrobot to counteract strong pulsatile flows or to navigate in small vessels, can be overcomed by using intra-arterial balloon or upsized magnetic microcarriers composed of aggregated nanoparticles, as illustrated in Fig. 1. Until now, the proposed closed-loop MRN strategies consider only simple Y-bifurcations [6] or when more complex phantoms are used, real-time imaging is based on MR-compatible vision cameras inserted in the MRI bore [10]. The design of a robust navigation strategy in complex phantoms should take into account the MRI technological constraints and physiological perturbations, e.g. non-negligible pulsatile flow, saturation of magnetic gradient amplitudes, limited duty cycle of imaging-propulsion sequences and MRI overheating [7]. To deal with these MRN constraints different navigation planning approaches have been proposed, such as using potential fields and the breadth-first search algorithm [6], or fast marching method (FMM) [14] considering the centerline [7], the uncertainty [11] or the power efficiency [15] objectives. However, each proposed navigation strategy does not solve all above constraints together. That is why, these aspects should be anticipated at the early navigation planning stage [15]. In this paper, we address these conflicting multi-objective issues by proposing a robust and autonomous navigation design based on a Pareto optimality framework.

The paper is organized as follows. The MRN system with its basic components (including sensing and actuation modules) are first introduced in Section II. Then, Section III presents the optimal navigation planning frameworks that allows dealing with multiple-objective problems. In Section IV some preliminary experiments are conducted to evaluate the MRN functionalities capabilities. Based on this preoperative results, an autonomous 2D MRN on an in vitro vascular phantom result is presented in Section V, validating the proposed MRN.

\section{TheORETICAL BACKGROUND}

\section{A. MRI Propulsion}

The overall concept of the magnetic resonance navigation $(\mathrm{MRN})$ relies on the use of a clinical magnetic resonance

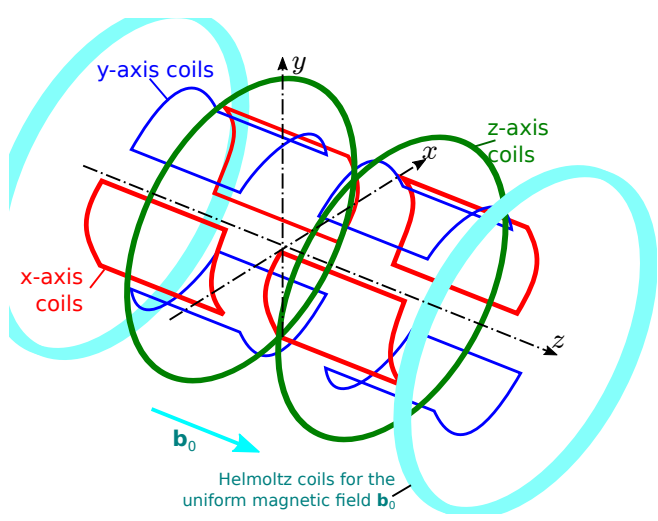

Fig. 2. Representation of the MRI magnetic gradient coils set configuration: $x$ and $y$ axes are pairs of saddle coils and $z$-axis are Maxwell coils pair.

imaging (MRI) scanner to both track and propel a therapeutic agent (termed microrobot). Basically, as depicted in Fig. 2, a MRI scanner comprises one pair of Helmholtz coils generating a strong static uniform magnetic field $\mathbf{b}_{0}$, and is aligned to the symmetry $z$-axis of the MRI bore. In order to generate the propulsion force of the microrobot, a magnetic gradient field in the 3D-space of the MRI bore is generated by two pairs of Golay coils and one pair of Maxwell coils in an orthogonal arrangement. The magnetic field $\mathbf{b}$ is governed by the following Maxwell equations:

$$
\nabla \times \mathbf{b}_{g}(r)=0 ; \nabla \cdot \mathbf{b}_{g}(r)=0
$$

This coil configuration is axis-symmetric and generates (at the center of the MRI bore) a magnetic field given by :

$$
\mathbf{b}_{g}=\left(\begin{array}{c}
-0.5 g_{z} x+g_{x} z \\
-0.5 g_{z} y+g_{y} z \\
g_{x} x+g_{y} y+g_{y} y
\end{array}\right)
$$

where

$$
\nabla \mathbf{b}_{g}=\left(\begin{array}{lll}
g_{x} & g_{y} & g_{z}
\end{array}\right)^{T}=\left(\begin{array}{lll}
\frac{\partial b_{x}}{\partial z} & \frac{\partial b_{y}}{\partial z} & \frac{\partial b_{z}}{\partial z}
\end{array}\right)^{T}
$$

are the linearly independent magnetic gradients. The $x, y, z$ coordinates originate at the center of the MRI bore where $z$ is the direction of the bore axis of symmetry. Besides the high magnetic field, uniform and linear magnetic gradients can be created within a 40-to-60-cm diameter spherical volume. For instance, the Magnetom $\left.{ }^{(}\right)$Verio $3 \mathrm{~T}$ is capable to generate an uniform magnetic field of $\mathbf{b}_{0}=3 \mathrm{~T}$ in the $z$-axis direction, and a magnetic gradient magnitude up to $45 \mathrm{mT} \mathrm{m}^{-1}$ in the $x$-y-z-axes directions. Placed in the MRI bore, the magnetic material volume $V_{\mathrm{m}}$ of a microrobot is subject to a total magnetic field $\mathbf{b}=\mathbf{b}_{0}+\mathbf{b}_{g}$. In such strong magnetic field, the magnetic material reaches the saturation magnetization $\mathbf{m}_{\text {sat }}=\left(0,0, m_{s z}\right)^{T}$, and is mainly aligned with $\mathbf{b}_{0}$, as $\mathbf{b}_{g} \ll \mathbf{b}_{0}$. In this study, only spherical beads are considered, and thus, only a magnetic force $\mathbf{f}_{\mathrm{m}}$ is induced, and is expressed here as:

$$
\mathbf{f}_{\mathrm{m}}=V_{\mathrm{m}} m_{s z} \nabla \mathbf{b}_{g},
$$

Only $\mathbf{b}_{g}$ contributes to the force generation since $\mathbf{b}_{0}$ is homogeneous and its spatial variation is zero. The equation states 
that three orthogonal magnetic forces act on the magnetic microrobot. This means that magnetic gradients of the MRI could induce 3 -DOF propulsion only in the center of the bore, within a workspace of approximately $40 \times 40 \times 40 \mathrm{~cm}^{3}$.

\section{B. Endovascular Modeling}

The modeling of the microrobot immersed in a microfluidic environment is necessary for the both navigation planning and control strategies. This modeling is illustrated in Fig. 3, and the corresponding dynamic model is briefly presented hereafter.

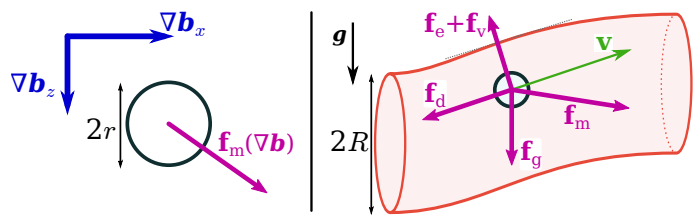

Fig. 3. Representation of the magnetic microrobot (left) in microfluidic environment (right) with the applied forces.

Actuated by magnetic gradients $\nabla \mathbf{b}_{g}$ in a microfluidic environment, the microrobot dynamics could be expressed as:

$$
(\rho V) \dot{\mathbf{v}}=\sum \mathbf{f}=\mathbf{f}_{\mathrm{m}}\left(\nabla \mathbf{b}_{g}\right)+\mathbf{f}_{\mathrm{Ext}}(\mathbf{v}, \mathbf{x})
$$

with $\rho, V$ and $\mathbf{v}$ the microrobot density, volume, and velocity respectively. $\mathbf{f}_{\mathrm{Ext}}$ are all external forces such as the apparent weight $\left(\mathbf{f}_{g}\right)$, electrostatic $\left(\mathbf{f}_{\mathrm{e}}\right)$, van der Waals $\left(\mathbf{f}_{\mathrm{v}}\right)$ and hydrodynamic drag $\left(\mathbf{f}_{\mathrm{d}}\right)$ microforces (see Fig. 3). At the considered scale, and when the microrobot navigates close to the centerline, it has been shown that the most dominant external force is the hydrodynamic drag force $\mathbf{f}_{\mathrm{d}}$ [15], [16]. In such case, a spherical microrobot of radius $r$ will then experience mainly the magnetic pulling (4), and the drag forces, that are commonly defined as:

$$
\mathbf{f}_{\mathrm{d}}=-6 \pi \eta r \cdot \mathbf{v}
$$

where $\eta$ is the flow viscosity.

Let $(\mathbf{v}, \mathbf{x})^{T}$ denotes the system state vector, defined by the microrobot velocity $\mathbf{v}$ and location $\mathbf{x}$. This position $\mathbf{x}$ is measured thanks to the developed real-time artifact tracking procedure [11]. The input vector is defined by the magnetic gradient, that is $\mathbf{u}=\nabla \mathbf{b}_{g}$. From the above dynamic model we get the following state-space representation $\left(\mathcal{S}_{\nabla}\right)$ :

$$
\left(\mathcal{S}_{\nabla}\right)=\left\{\begin{array}{c}
\left(\begin{array}{c}
\dot{\mathbf{x}} \\
\dot{\mathbf{v}}
\end{array}\right)=\mathbf{A}\left(\begin{array}{c}
\mathbf{x} \\
\mathbf{v}
\end{array}\right)+\mathbf{B u}+\left(\begin{array}{c}
\Delta \gamma_{c} \\
\Delta \gamma_{p}
\end{array}\right) \\
\mathbf{y}=\mathbf{C} \mathbf{x}+\Delta \gamma_{b}
\end{array}\right.
$$

with

$$
\mathbf{A}=\left(\begin{array}{cc}
0 & \mathbb{I}_{2} \\
0 & -4.5 \frac{\eta}{\rho r^{2}} \mathbb{I}_{2}
\end{array}\right) ; \mathbf{B}=\left(\begin{array}{c}
0 \\
\frac{m_{s z} \tau_{m}}{\rho} \mathbb{I}_{2}
\end{array}\right) ; \mathbf{C}=1
$$

and $\mathbb{I}_{2}$ the identity matrix, and $\tau_{m}=V_{\mathrm{m}} / V$ the volumetric magnetization rate.

The system $\left(\mathcal{S}_{\nabla}\right)$ models the robot dynamics, and has to be controlled along a reference trajectory subjected to uncertainty errors. The terms $\Delta \gamma_{c}, \Delta \gamma_{p}$ and $\Delta \gamma_{b}$ represent the uncertain parameters related to the influence of operating conditions, i.e. the external forces $\mathbf{f}_{\mathrm{Ext}}$ affecting the microrobot's dynamics [16], the saturation of the MRI actuator coils [10] and the 2-D position measurement errors related to MRI magnetic signature sensing [11], respectively. Because safety and accuracy are of critical importance, these uncertainties will have significant influence on the choice of the best path for MRN procedure. Different works show how to incorporate uncertainty and state uncertainty into the control framework. As example, predictive controller [17], adaptive controller [18] or $H_{\infty}$ controller [19] proved to be efficient as proof of concept but fail when considering multiple uncertainties in realistic and complex vascular navigation configurations under pulsatile flow.

The challenge we discuss in the following section is to precisely quantify these uncertainties in advance, such as the best path can be selected in realistic MRN conditions.

\section{OPTIMAL MRI-BASED NAVIGATION FRAMEWORK}

\section{A. Navigation Planning}

1) Problem Statement: A navigation path between a starting point $\mathbf{x}_{0} \in \mathcal{C}$ to a targeted point $\mathbf{x} \in \mathcal{C}$, could be defined as a curve $\mathbf{p}$ on the domain $\mathcal{C} \subset \mathbb{R}^{d}$ as follows:

$$
\mathbf{p}: l \in[0,1] \mapsto \mathbf{p}(l) \in \mathcal{C}, \text { with } \mathbf{x}_{0}=\mathbf{p}(0), \mathbf{x}=\mathbf{p}(1)
$$

where $d=2,3,4 \ldots$ is the path state-space dimension, and $l$ the arc-length parameter. In a Riemann manifold the path length could be defined from an energy functional weighted by a metric $w: \mathbf{x} \in \mathcal{C} \mapsto w(\mathbf{x}) \in \mathbb{R}^{+}$as follows:

$$
L_{w}(\mathbf{p})=\int_{0}^{1} w(\mathbf{p}(l)) \cdot\|\dot{\mathbf{p}}(l)\| d l
$$

with $\|\cdot\|$ the Euclidean norm. Classically, the navigation planning goal is to find a minimal path (also referred as a geodesic) that globally minimizes the path length $L_{w}(\mathbf{p})$, that is:

$$
\gamma=\underset{\mathbf{p}}{\arg \min } L_{w}(\mathbf{p})
$$

The geodesic distance between two points $\left(\mathbf{x}_{0}, \mathbf{x}\right)$ is the length $L_{w}(\gamma)$ wrt. the metric $w$. In this context, previous studies [11], [15], [17] have shown that the fast marching method (FMM), introduced by Sethian [14], offers an interesting framework.

2) Fast Marching Path Planning: In the FMM framework, the minimum path finding problem (11) is solved through the computation of the minimal distance map $\mathcal{U}: \mathcal{C} \mapsto \mathbb{R}^{+}$using the Eikonal equation:

$$
\begin{cases}\|\nabla \mathcal{U}(\mathbf{x})\| & =w(\mathbf{x}), \quad \forall \mathbf{x} \in \mathcal{C} \\ \mathcal{U}\left(\mathbf{x}_{0}\right) & =0\end{cases}
$$

The key issue of the FMM is to define an appropriate metric $w(\mathbf{x})$ for retrieving a suitable navigation path based on the desired criteria.

3) Minimal Centerline: A first objective is to design the metric on spatial consideration. A basic idea is to use a unit metric, where $w_{1}(\mathbf{x})=1$ for all free location in the domain $\mathcal{C}$, and 0 in forbidden location. Such unit metric computes from Eq. (10) the Euclidean path arc-length $L_{1}=L_{w_{1}}\left(\gamma_{1}\right)$, and thus leads to the shortest path. However, this solution 
commonly implies contouring the walls. In previous work [17] the following spatial cost function has been proposed:

$$
w_{\mathrm{c}}: \mathbf{x} \in \mathcal{C} \mapsto \operatorname{Enhance}(\mathbf{x}) \in \mathbb{R}^{+}
$$

where Enhance $(\mathbf{x})$ is a vesselness enhancement function designed to give their maximal response in the center of the thoroughfare. Such filtering technique leads to find the centerline geodesic $\gamma_{c}$ [17]. In particular, it has been shown that the microrobot dynamics is less affected by the surface forces (the contact, electrostatic and van der Waals microforces) when it navigates close to the centerline [15], [16]. For such objective, $\gamma_{c}$ is then an optimal solution. However, designed solely on spatial consideration, the centerline geodesic does not take into account neither the physiological perturbations, the MRI technological constraints nor the sensing uncertainties.

4) Minimum-Power Navigation Path: To ensure that a geodesic is really achievable by the MRN system the navigation path has to reduce the maximum pulling gradients (e.g. up to $80 \mathrm{mT} \mathrm{m}^{-1}$ ), the operating time (e.g. $\leq 30 \mathrm{~min}$ ), and also maintain the rising temperature of the electromagnetic coils within operating limits (e.g. $\leq 50{ }^{\circ} \mathrm{C}$ ) [10]. To overcome such issues, in [15] an anisotropic cost function is proposed to minimize the energy expenditure, and is expressed as:

$$
w_{\mathrm{p}}:(\mathbf{x}, \mathbf{f}) \in \mathcal{C} \times \mathcal{F} \mapsto w_{\mathrm{p}}(\mathbf{x}, \mathbf{f}) \in \mathbb{R}^{+}
$$

where the anisotropy cost is extended with the vector $\mathbf{f}$ of a field of force $\mathcal{F}$. The proposed idea is to use a classical power functional expression [15]:

$$
w_{\mathbf{p}}(\mathbf{x}, \mathbf{f})=\underset{\mathbf{x} \in \mathcal{C}, \nabla \mathbf{b} \in \mathcal{B}}{\arg \max }\langle\mathbf{f}(\mathbf{x}, \nabla \mathbf{b}) \cdot \mathbf{v}(\mathbf{x})\rangle
$$

where $\langle\cdot\rangle$ is the inner product of $\mathbb{R}^{d}$, and $\mathbf{f}(\mathbf{x}, \nabla \mathbf{b})$ is the applied resultant force that embeds the controlled magnetic gradient $\nabla \mathbf{b} \in \mathcal{B}$. Using model of the system dynamics (7), the anisotropic metric $w_{\mathrm{p}}$ allows considering the major physiological perturbations (e.g. flow viscosity, time-varying pulsatile flow). Furthermore, the magnetic action set $\mathcal{B}$ is designed wrt. the MRI available magnetic actuation capabilities. The metric $w_{\mathrm{p}}$ allows obtaining a power efficient geodesic $\gamma_{\mathrm{p}}$ effectively feasible by the MRN platform.

5) Planning with MRN Uncertainties: Numerous sources of uncertainties, such as in MRI data acquisition and processing, carrier model and position tracking, must be considered in MRN planning. Otherwise, the microrobot may be trapped in the wrong bifurcation or cause some unwanted embolies. Explicitly modeling sensing uncertainties in the planning strategy is a first main step to robustness [11]. It has been recognized that it could be defined some maps which describe the quality of the sensed information obtained at each configuration used by the system [20], [21]. In previous study [11], the expected information gain $\mathcal{I}(\mathbf{x} ; \mathbf{y})$ is proposed to design this mapping, and the corresponding metric is defined by

$$
w_{\mathrm{b}}: \mathbf{x} \in \mathcal{C} \mapsto \mathcal{I}^{-1}(\mathbf{x} ; \mathbf{y}) \in \mathbb{R}^{+}
$$

The expected information gain quantifies the microdevice's ability to characterize its localization at different positions $\mathbf{x}$. $\mathcal{I}(\mathbf{x} ; \mathbf{y})$ is computed, given an observed data $\mathbf{y}$, from the difference in Shannon entropy of the prior and posterior distributions: $\mathcal{I}(\mathbf{x} ; \mathbf{y})=H(\mathbf{x})-H(\mathbf{x} \mid \mathbf{y})$. The prior entropy $H(\mathbf{x})$ measures the carrier's belief of its position $\mathbf{x}$, before the sensory input $\mathbf{y}$ is received. The conditional entropy $H(\mathbf{x} \mid \mathbf{y})$ denotes the expected entropy change after measurement data $\mathbf{y}$. The computed geodesic $\gamma_{\mathrm{b}}$ from the metric $w_{\mathrm{b}}(16)$ maximizes the expected information gain $\mathcal{I}(\mathbf{x} ; \mathbf{y})$, and thus minimizes the uncertainties along the navigation path.

\section{B. Navigation Planning with Multiple Objectives}

Previous proposed solutions provide an optimal navigation pathway wrt. their corresponding metric. The considered navigation planning strategy is then a basic single-objective optimization problem (11). When more than one goal has to be addressed, the design problem becomes multi-objective. In this context, a geodesic is commonly obtained from:

$$
\gamma=\underset{\mathbf{p}}{\arg \min }\left\{L_{w_{1}}(\mathbf{p}), \ldots L_{w_{k}}(\mathbf{p}), \ldots L_{w_{n}}(\mathbf{p})\right\}
$$

As the different metrics $w_{k}$ are usually conflicting, the scalar concept of "optimality" does not apply directly in the multiobjective design. This situation gives rise to the concept of a Pareto solution, and the use of Pareto optimality. Especially, a feasible path $\mathbf{p}^{\star}$ is said to be Pareto optimal for a multiobjective problem if there are no other feasible path $\mathbf{p}$ such that: $L_{w_{k}}\left(\mathbf{p}^{\star}\right) \leq L_{w_{k}}(\mathbf{p}), \forall k=1 . . n$, and $L_{w_{k}}\left(\mathbf{p}^{\star}\right)<L_{w_{k}}(\mathbf{p})$ for at least one $k=1$..n. The family of Pareto solutions forms the so-called Pareto front.

The most widely-used approach for multi-objective optimization is the weighted sum technique (also referred as scalarization method), where multiple metrics are aggregated in a single cost function as follows [22]:

$$
w_{\lambda}=\sum_{k=1}^{n} \lambda_{k} w_{k}
$$

If $\sum_{k} \lambda_{k}=1$ and $\forall \lambda_{k} \in[0,1]$ the weighted sum is said to be a convex combination of cost functions. Any weight set $\left\{\lambda_{k}\right\}$ generates a single cost function $w_{\lambda}$ that determines different Pareto optimal geodesics. In particular, minimizing (18) is sufficient for Pareto optimality [22]. Testing all possible values of the weight set would construct the convex hull of the Pareto front. It can be shown that the navigation path computed from the single-cost function $w_{\lambda}$ is a Pareto optimum geodesic for the original multi-objective problem (17).

However, usually there is no correspondence between a set $\left\{\lambda_{k}\right\}$ and a geodesic: it is up to the end-user to choose the appropriate weights. Expert knowledge is then needed to select the weights while the conditions of the system changed. To propose a weight set, an idea is to use the utopian objective (also referred as ideal point) defined by:

$$
L^{\star}=\inf _{\mathbf{p}} L_{w_{k}}(\mathbf{p}), \forall k=1 . . n
$$

Nevertheless, this utopian objective $L^{\star}$ is often unattainable as the costs are conflicting. Next idea is to consider the set $\left\{\lambda_{k}\right\}$ that generates the closest solution to utopian $L^{\star}$. Such approach leads to seek for the Pareto optimal compromise geodesic:

$$
\widehat{\gamma}=\underset{\mathbf{p},\left\{\lambda_{k}\right\}}{\arg \min }\left\|L_{w_{\lambda}}(\mathbf{p})-L^{\star}\right\|
$$




\section{Preoperative Experimental Results}

\section{A. Experimental Conditions}

The experiments are conducted using a clinical Magnetom ${ }^{\circledR}$ Verio 3T MRI scanner (Siemens, Germany) providing realtime capabilities. Magnetic microrobots are made of steel bead of different sizes. They are placed either in an acrylic box for sensing characterization, or in a vascular phantom for MRN evaluation. The phantom is made of two PMMA plates thermally bonded together, and has been divided into different channels with multiple bifurcations. The general characteristics are given in table I. The vascular phantom was filled with deionized water, and placed inside the linear gradient volume of the MRI bore on the $x-z$ (coronal) plane, as shown in Fig. 4. The Table II summarizes the relevant parameters considered in the experiments.

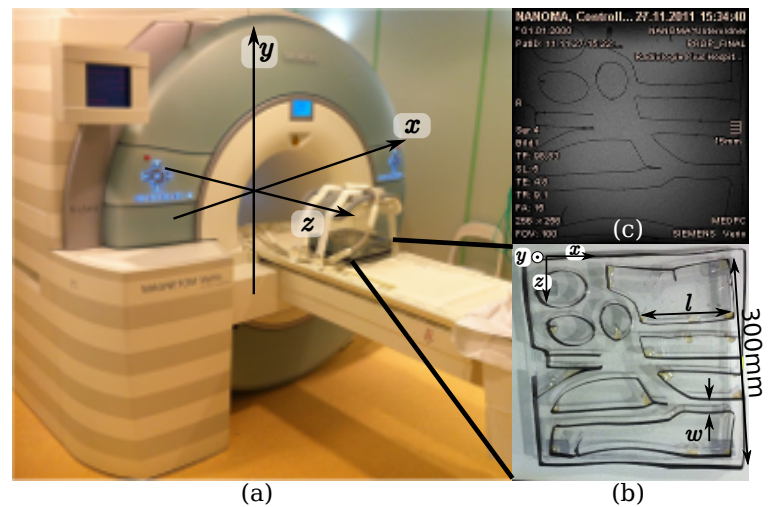

Fig. 4. Experimental setup that comprises: (a) Magnetom Verio 3T MRI scanner (Siemens, Germany) with the acrylic box which contains the vascular phantom (b). The inset (c) illustrates a typical MR-imaging of the phantom.

TABLE I

GEOMETRIC CHARACTERISTICS OF THE VASCULAR PHANTOM.

\begin{tabular}{cccc}
\hline Size $(\mathrm{mm})$ & $w(\mathrm{~mm})$ & $l(\mathrm{~mm})$ & Depth $(\mathrm{mm})$ \\
\hline $300 \times 300$ & $8.1-12.3$ & $30-300$ & 6.0 \\
\hline
\end{tabular}

TABLE II

EXPERIMENTAL CONDITIONS.

\begin{tabular}{|c|c|c|c|}
\hline & \multicolumn{2}{|l|}{ Parameter } & Values \\
\hline \multirow{3}{*}{$\begin{array}{l}\text { Microrobot } \\
\text { (steel ball) }\end{array}$} & & $r$ & $750 \mu \mathrm{m}$ \\
\hline & density & $\rho$ & $7850 \mathrm{~kg} \mathrm{~m}^{-3}$ \\
\hline & magnetization & $m_{s z}$ & $1.6 \times 10^{6} \mathrm{~A} \mathrm{~m}^{-1}$ \\
\hline Water & viscosity & $\eta$ & $1.005 \times 10^{-3} \mathrm{Pas}$ \\
\hline
\end{tabular}

Fig. 5 illustrates the gradient pulse generated during a propulsion step in the $x$-direction followed by a slice acquisition using a fast low angle shot (FLASH) sequence. The propulsion gradient is set to $\nabla \mathbf{b}_{g}=(20,0,0)^{T} \mathrm{mT} \mathrm{m}^{-1}$ for $T_{\text {Prop }}=640 \mathrm{~ms}$. The acquisition parameters are a repetition time of $T_{r}=7.5 \mathrm{~ms}$ and echo time of $T_{e}=4.8 \mathrm{~ms}$, with an image resolution of $256 \times 256$ and a halfed $\mathrm{k}$-space, leading to an acquisition time of $T_{\mathrm{Acq}}=960 \mathrm{~ms}$.

To magnetically propel the microrobot to its destination, different control strategies could be considered such as simple
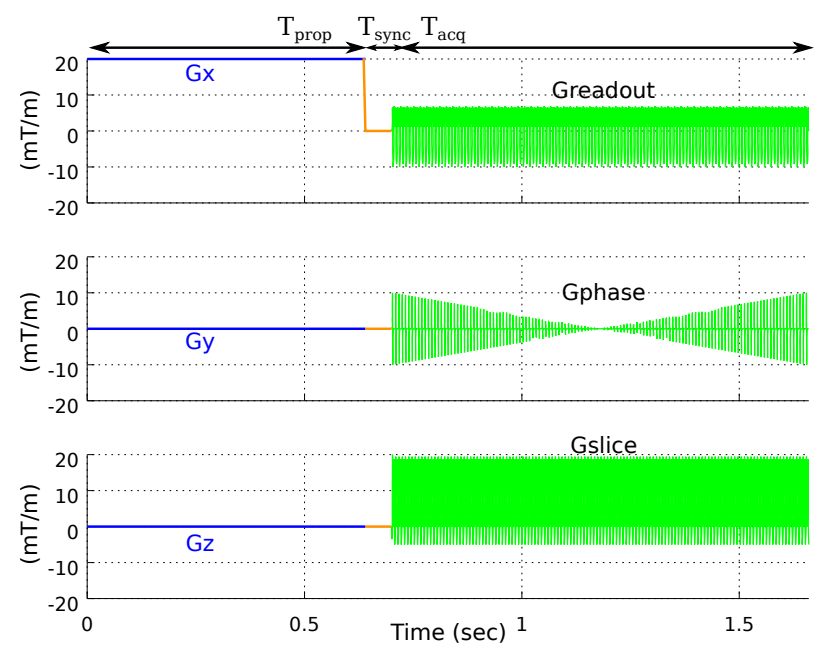

Fig. 5. Magnetic gradient sequences for propulsion and acquisition during one time cycle $T_{\text {Cycle. The }} x-y-z$ magnetic gradient components during the propulsion $\left(T_{\text {Prop }}\right)$, synchronization $\left(T_{\text {Sync }}\right)$ and acquisition $\left(T_{\text {Acq }}\right)$ sequences are reported.

proportional-integral-derivative (PID) [6], adaptive backstepping control schemes [18] or predictive [17]. The capabilities of the MRI scanner to actuate properly a given magnetic microrobot are related to different factors. First, the duty cycle $\tau=\frac{T_{\text {Prop }}}{T_{\text {Cycle }}}$ of the time multiplexing scheme directly influences the microrobot motion and then the controller performance. In a previous study [17], we have shown that an optimum duty cycle is obtained for $\tau=40 \%$, which is similar to the one reported in [6]. The microrobot magnetic properties also influence the MRI propulsion capabilities through the magnetic force given by Eq. (4). Moreover, the imaging part of the MRI sequence also produces magnetic gradient pulses which do not necessarily sum up to zero. For the previous example depicted in Fig. 5, the mean magnetic gradient values are $(31.20,0,0)^{T} \mathrm{mT} \mathrm{m}^{-1}$ for the propulsion phase $T_{\text {Prop }}$, whereas during the imaging phase $T_{\text {Acq }}$ it was $(-2.49,0.21,1.65)^{T} \mathrm{mT} \mathrm{m}^{-1}$. The gradient fields generated during the sensing phase does not appear negligible. However, as the imaging sequence does not change significantly during the overall navigation process, the induced force can be treated as a constant external perturbation in the control scheme.

\section{B. MRI Sensing Capability}

Here, to compute the Shannon entropy $H(\mathbf{x} \mid \mathbf{y})$, we assume that the sensing process could be modeled by a white bivariateGaussian distribution: $\mathcal{N}\left(0, \Sigma_{\mathbf{y}}\right)$, with zero mean and $\Sigma_{\mathbf{y}}$ the covariance matrix (see [11]). This covariance matrix is computed from the standard deviation considering:

$$
\Sigma_{\mathbf{y}}=\operatorname{diag}\left(\sigma_{x}^{2}, \sigma_{z}^{2}\right)
$$

where $\sigma_{x}$ and $\sigma_{z}$ are the standard deviations along the $x$ and $z$-axis respectively. To consider microrobots of any size, it is necessary to develop an analytical model of the covariance matrix that fits the measured sensing capability (see [11]). To do so, Fig. 6 shows a third order polynomial interpolation fitting suitably the measured standard deviations (circle and 


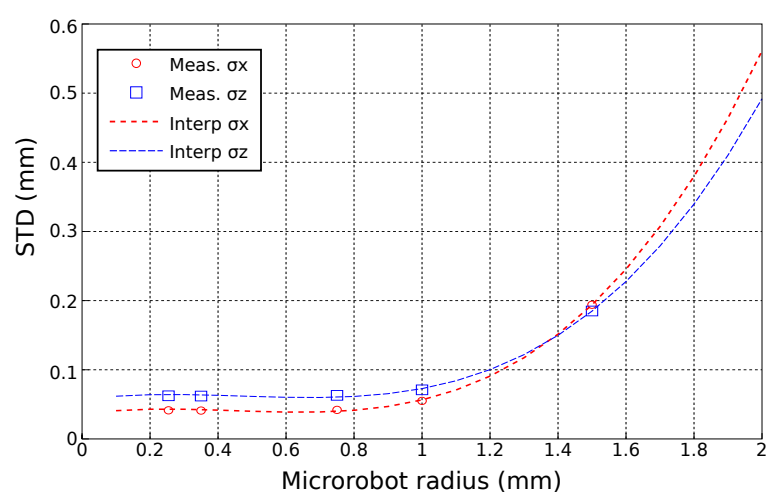

Fig. 6. Polynomial interpolation of the standard deviations (std) of the MRtracking of steel ball microrobots using a FLASH sequence.

square markers) wrt. the microrobot size. It should be noticed that the standard deviations scale favorably until to reach a low plateau value when the microrobot size is below $1 \mathrm{~mm}$.

\section{MRI Steering Capability Evaluation}

To evaluate the MRN propulsion capability, an imaging and propulsion sole sequence is applied on a spherical microrobot with a radius of $r=750 \mu \mathrm{m}$. As in Section II-A, the steering gradient has been set to $\nabla \mathbf{b}=(20,0,0)^{T} \mathrm{mT} \mathrm{m}^{-1}$ for $T_{\text {Prop }}=640 \mathrm{~ms}$. Similarly, the sensing process uses the same optimized FLASH sequence as it provides a good compromise in terms of accuracy and acquisition time. Fig. 5 depicts the applied magnetic gradient sequence for propulsion and acquisition for one cycle time $T_{\text {Cycle }}$. Fig. 7 shows the corresponding velocities of the magnetic microrobot that has traveled a distance of $243.4 \mathrm{~mm}$ during $T_{\text {Cycle }}$. Moreover, during the acquisition step, the velocity of the microrobot does not appear to be affected by the MR-imaging sequence. This result demonstrates the effectiveness of the tracking and propulsion sequence of a magnetic microrobot through a clinical MRI scanner.

\section{Preoperative MRN Planning}

1) Single Objective Navigation Planning: The proposed magnetic resonance navigation (MRN) planning framework has been first evaluated considering each presented single objective. More precisely, the goal here is to retrieve the centerline, $\gamma_{c}$, power efficient, $\gamma_{p}$, and the belief, $\gamma_{b}$, geodesics. Furthermore, the sensing uncertainty model is designed upon FLASH sequences as it provides the best sensing capabilities.

Firstly, a highly magnetic stain-less steel bead microrobot with a radius of $r=750 \mu \mathrm{m}$ is assumed to navigate in the vascular phantom filled with water (ie., a static flow), with the experimental condition parameters summarized in Table II. The corresponding metric maps $\left(w_{\mathrm{c}}, w_{\mathrm{p}}\right.$ and $\left.w_{\mathrm{b}}\right)$ are calculated and are illustrated in Fig.8. Fig.9a illustrates a navigation case starting from $\mathbf{x}_{03}$ and ending to $\mathbf{x}_{3}$ locations. Table III presents the geodesic lengths $L_{w}(\gamma)$, computed from Eq (10), for each navigation path. considering a unit (i.e., that allows retrieving the Euclidean path arc-length $L_{1}$ ), centerline

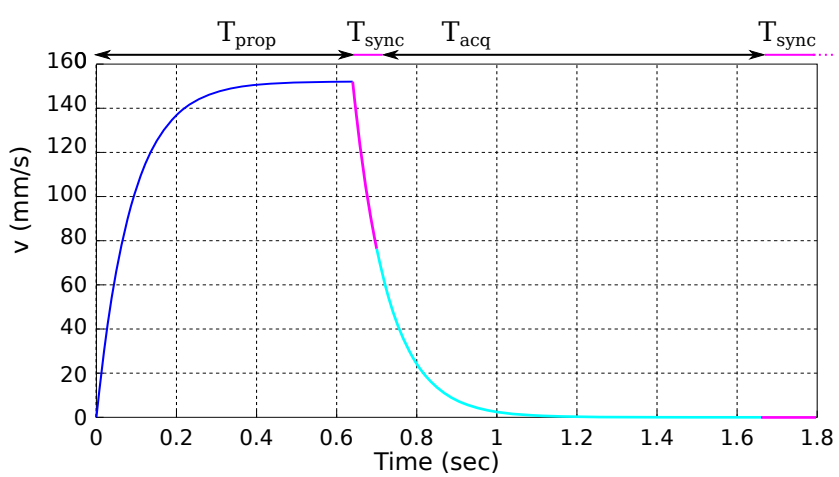

(a)

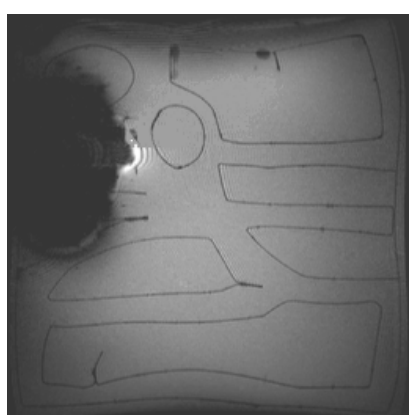

(b)

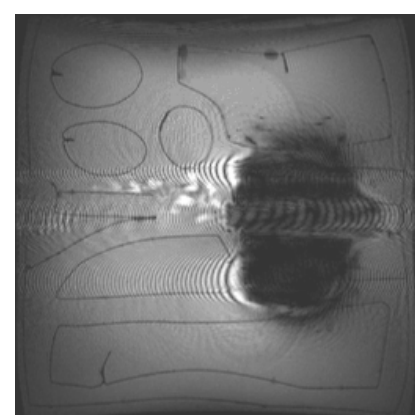

(c)
Fig. 7. Time-multiplexing and MRI-based actuation: (a) velocities of a spherical magnetic microrobot with a radius $r=750 \mu \mathrm{m}$; (b) initial and (c) final position of the magnetic microrobot.

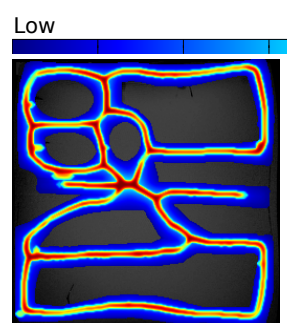

(a)

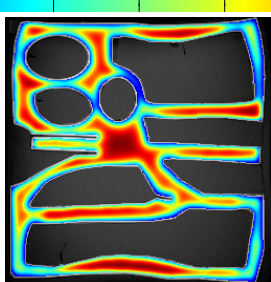

(b)

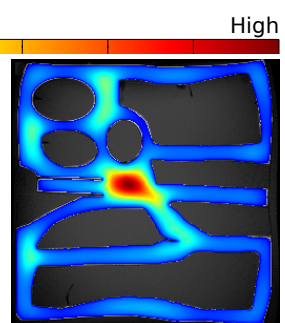

(c)
Fig. 8. The calculated metrics: (a) the centerline $\left(w_{\mathrm{c}}\right)$; (b) the power $\left(w_{\mathrm{p}}\right)$; and $(\mathrm{c})$ the sensing uncertainties $\left(w_{\mathrm{b}}\right)$.

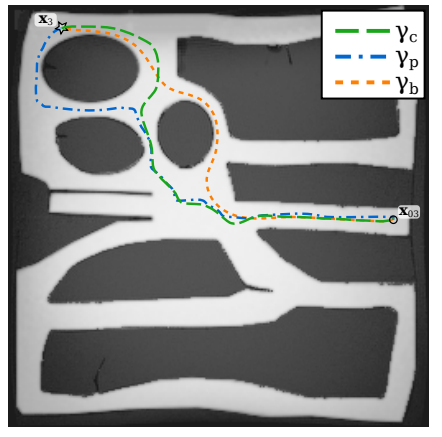

(a)

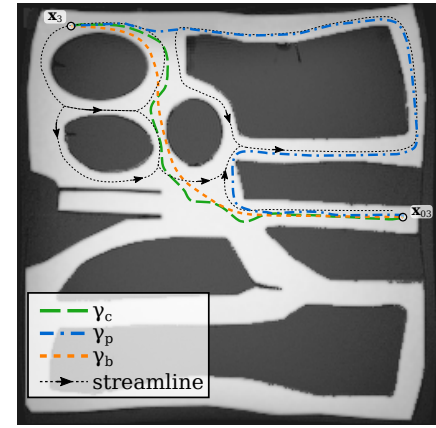

(b)
Fig. 9. Optimal navigation paths with the centerline $\left(\gamma_{c}\right)$, the power efficient $\left(\gamma_{\mathrm{p}}\right)$, and the belief $\left(\gamma_{\mathrm{b}}\right)$ metrics. (a) A steel ball with a radius of $r=750 \mu \mathrm{m}$ navigating in a static flow; and (b) a steel ball with a radius of $r=1500 \mu \mathrm{m}$ in a continuous flow with a velocity $\|\mathbf{v}\|=20 \mathrm{~mm} \mathrm{~s}^{-1}$.

$w_{\mathrm{c}}$, power $w_{\mathrm{p}}$, and belief $w_{\mathrm{b}}$ metrics. As expected, each 


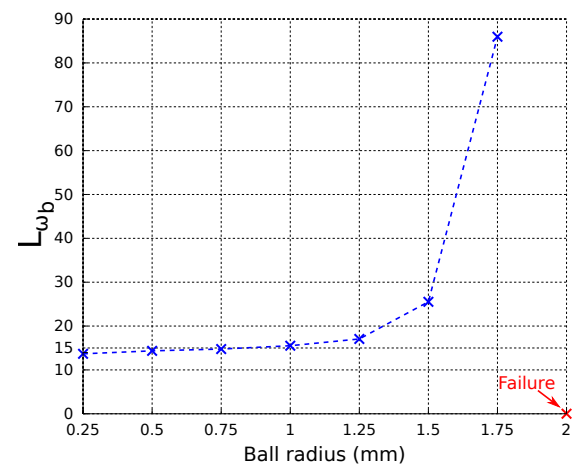

(a)

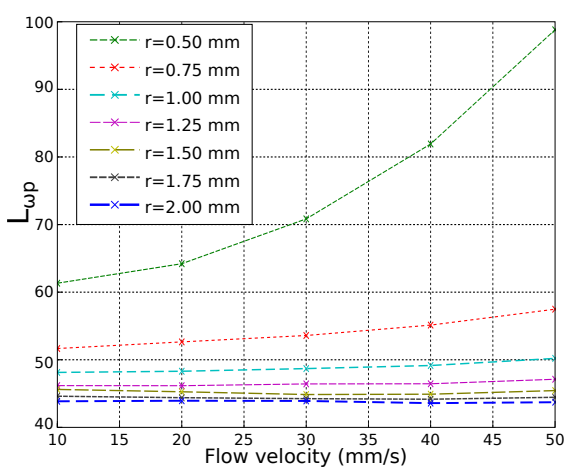

(b)

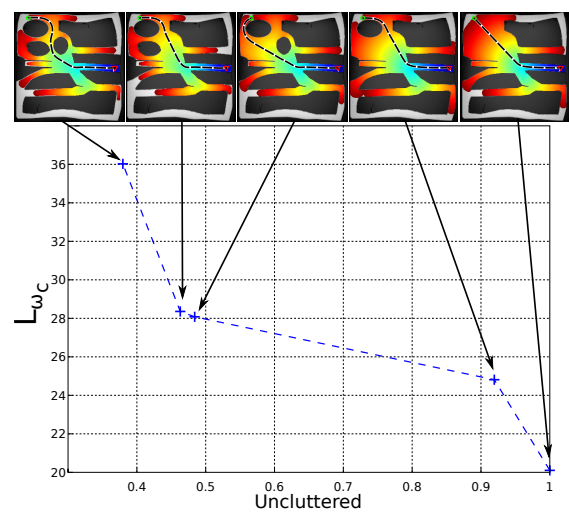

(c)

Fig. 10. Evolution of the metrics: (a) belief energy functional $L_{w_{\mathrm{b}}}$ as function of the steel ball radius. (b) Power efficiency energy functional $L_{w_{\mathrm{p}}}$ as function of the flow magnitude for different steel ball radii. (c) Centerline efficiency energy functional $L_{w_{\mathrm{p}}}$ as function of the uncluttered environment.

geodesic provides the minimal length with its own metric against the others objective functions. In the case illustrated in Fig.9b, the geodesics are calculated when a $r=1500 \mu \mathrm{m}$ steel bead microrobot is navigating in presence of a steady flow $\left(\|\mathbf{v}\|=20 \mathrm{~mm} \mathrm{~s}^{-1}\right)$ simulating the arterial blood velocity. Fig.9b depicts the relevant streamline of this virtual flow. As expected, solely based on spatial information, the centerline, $w_{\mathrm{c}}$, metric gives exactly the same solution as the previous case. In contrast, the belief, $\gamma_{\mathrm{b}}$, and the power efficient, $\gamma_{\mathrm{p}}$, geodesics provide new solutions. It should be noticed that the change of the size of the microrobot leads to a change of the computed information gain which is related to the standard deviation of the MRI sensing process (see also Fig. 6), together with a change in its location belief. Actually, when a larger microrobot is considered the environment uncertainty is increasing, especially in smaller channels. Considering this, we investigated experimentally the evolution of the energy functional $L_{w_{\mathrm{b}}}$ as function of the microrobot's size (Fig.10a). The belief geodesic $\gamma_{\mathrm{b}}$ solution scales down favorably for robot sizes less than $1 \mathrm{~mm}$ but is strongly limited for robot sizes bigger than $2 \mathrm{~mm}$ due to the strong artifact signature. The circulating fluid flow plays an important role on the power geodesic, $\gamma_{\mathrm{p}}$. The navigation pathway tends to follow the circulating flow in order to minimize the energy expenditure. As illustration, Fig. 10b shows the power efficiency energy functional, $L_{w_{\mathrm{p}}}$ with respect to the flow magnitude for different microrobot sizes. As the robot size scales down, the required power increases drastically in presence of fluid flow failing to find a geodesic, $\gamma_{\mathrm{p}}$, solution for flow velocities values greater than $50 \mathrm{~mm} \mathrm{~s}^{-1}$. The power efficiency energy functional $\gamma_{\mathrm{p}}$ values are found nearly constant for velocity values ranging from $10 \mathrm{~mm} \mathrm{~s}^{-1}$-to- $50 \mathrm{~mm} \mathrm{~s}^{-1}$ for robot sizes greater than $1 \mathrm{~mm}$. Finally, the euclidean shortest path shown in Fig.10c is generally obtained using the centerline efficiency energy functional, $\gamma_{c}$, as the corresponding geodesic is less sensitive to wall effects [15]. In conclusion, optimal navigation pathways can be predicted when considering a tradeoff between the efficiencies of different cost functions according to the corresponding MRN experimental conditions.

2) Multi-Objective Planning:
TABLE III

THE LENGTHS OF EACH GEODESICS CONSIDERING THE UNIT $\left(w_{1}\right)$, CENTERLINE $\left(w_{\mathrm{c}}\right)$, POWER $\left(w_{\mathrm{p}}\right)$ AND BELIEF $\left(w_{\mathrm{b}}\right)$ METRICS, FOR THE DIFFERENT CASES SHOWN IN FIG.9.

\begin{tabular}{cc|cccc} 
Cases & & $L_{1}(\mathrm{~mm})$ & $L_{w_{\mathrm{c}}}$ & $L_{w_{\mathrm{p}}}$ & $L_{w_{\mathrm{b}}}$ \\
\hline \hline Fig.9a & $\gamma_{\mathrm{c}}$ & 383.621 & $\mathbf{2 9 . 5 6 0}$ & 21.081 & 18.152 \\
\multirow{2}{*}{$\mathbf{x}_{03} \rightarrow \mathbf{x}_{3}$} & $\gamma_{\mathrm{p}}$ & 472.331 & 35.567 & $\mathbf{1 9 . 9 3 0}$ & 21.759 \\
& $\gamma_{\mathrm{b}}$ & $\mathbf{3 1 9 . 8 6 9}$ & 33.936 & 20.488 & $\mathbf{1 5 . 4 2 5}$ \\
\hline \multirow{2}{*}{ Fig.9b } & $\gamma_{\mathrm{c}}$ & 383.621 & $\mathbf{2 9 . 5 6 0}$ & 126.164 & 17.143 \\
\multirow{2}{*}{$\mathbf{x}_{03} \rightarrow \mathbf{x}_{3}$} & $\gamma_{\mathrm{p}}$ & 725.456 & 95.623 & $\mathbf{4 5 . 5 0 6}$ & 38.384 \\
& $\gamma_{\mathrm{b}}$ & $\mathbf{3 5 7 . 4 5 8}$ & 43.534 & 103.567 & $\mathbf{1 0 . 2 3 8}$ \\
\hline
\end{tabular}

a) Bi-objective Optimization: The simplest multiobjective case is to consider two objectives that have to be optimized. Using the weighted sum method, the cost functions are simply aggregated as follows:

$$
w_{\lambda}=(1-\lambda) w_{x}+\lambda w_{y}, \quad \forall \lambda \in[0 ; 1]
$$

with $w_{x}$ and $w_{y}$ one of proposed metric, that is $w_{\mathrm{c}}, w_{\mathrm{p}}$ or $w_{\mathrm{b}}$ (see also Table IV).

Fig. 12 shows the Pareto front between the considered cost functions. For the sake of consistent comparison between objectives, each geodesic length $L_{w}$ are here normalized. In particular, a cost of 1 is the worst solution whereas 0 leads to the best one. Fig. 12a presents the Pareto front related to the normalized length of geodesics starting from point $\mathbf{x}_{01}$ to targeted location $\mathbf{x}_{1}$ corresponding to the case (1) in Fig.11. Each bi-objective problem seems to provide a convex Pareto front, but with different curvatures. Recalling that all solutions on the Pareto frontier (the circles in Fig. 12) are optimal according to the objective $w_{\lambda}$ (22), one can choose any of them. These results show that objectives of centerline $w_{\mathrm{c}}$ and belief $w_{\mathrm{b}}$ have the most influence against power metric.

Moreover, in the case (2), $\mathbf{x}_{02} \rightarrow \mathbf{x}_{2}$, illustrated in Fig.11 objective $w_{\mathrm{c}}$ provides a different solution than other cost functions. The distinctive solutions appear clearly in Fig. 12b for centerline $w_{\mathrm{c}}$ vs. belief $w_{\mathrm{b}}$ with a jump in the Pareto front. This gap is due to the fact that the geodesic could be below (the group $\{1\}$ in Fig. 12b) or above (the group $\{2\}$ in Fig. 12b) the obstacle. For centerline $w_{c}$ vs. power 


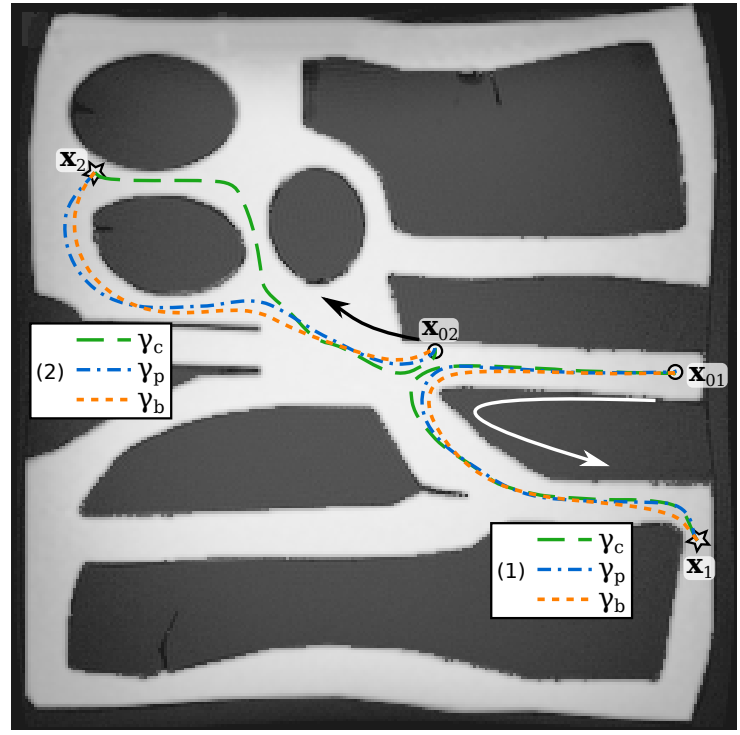

Fig. 11. Optimal navigation path with centerline $\left(\gamma_{c}\right)$, the power efficient $\left(\gamma_{\mathrm{p}}\right)$, and the belief $\left(\gamma_{\mathrm{b}}\right)$ metrics considering different starting $\left(\mathbf{x}_{01}, \mathbf{x}_{02}\right)$ and ending $\left(\mathbf{x}_{1}, \mathbf{x}_{2}\right)$ locations
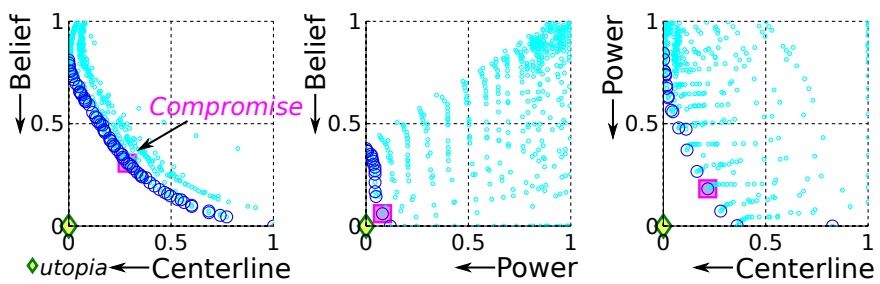

(a)
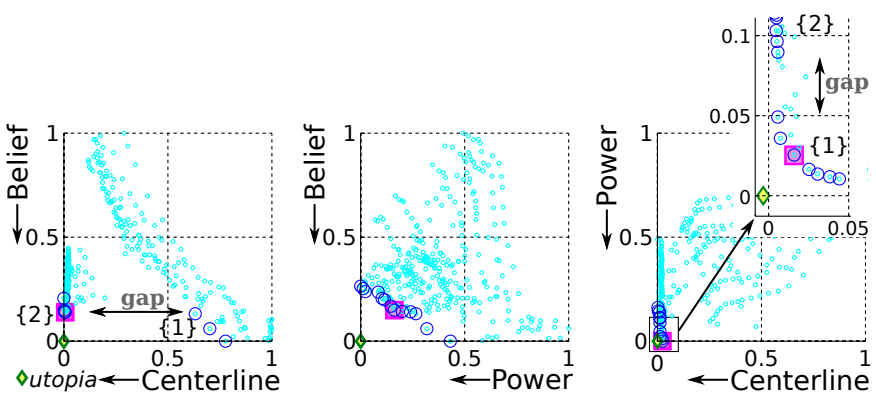

(b)

Fig. 12. Bi-objective Pareto Front: (left) centerline $w_{\mathrm{c}}$ vs. belief $w_{\mathrm{b}}$; (center) power $w_{\mathrm{p}}$ vs. belief $w_{\mathrm{b}}$; and (right) centerline $w_{\mathrm{c}}$ vs. power $w_{\mathrm{p}}$ objectives, from (a) $\mathbf{x}_{01}$ to $\mathbf{x}_{1}$ and (b) $\mathbf{x}_{02}$ to $\mathbf{x}_{2}$. Circles depict the Pareto nondominated solutions, and the square box the compromise solution wrt. the utopian solution (diamond).

$w_{\mathrm{p}}$ objectives, it is more difficult to characterize the switch between the solutions. In-depth analysis brings up a gap from a weight of $\lambda>0.7$, and is illustrated in the zooming inset in Fig. 12b. This kind of switch is also related to a non-convexity in the Pareto front. Moreover, as mentioned, among the Pareto non-dominated solutions, the compromise weight, $\hat{\lambda}$, is the closest to the utopia point $(0,0)$. Table IV summarizes the normalized length of the compromise navigation path. These solutions could offer a suitable choice as it entails minimizing the difference between potential optimal points.
TABLE IV

NORMALIZED LENGTH OF THE COMPROMISE GEODESIC FOR BI-OBJECTIVE OPTIMIZATION.

\begin{tabular}{cc|c|ccc} 
& $w_{\lambda}$ & $\widehat{\lambda}$ & $L_{w_{\mathrm{c}}}$ & $L_{w_{\mathrm{p}}}$ & $L_{w_{\mathrm{b}}}$ \\
\hline \hline (a) & $(1-\lambda) w_{\mathrm{c}}+\lambda w_{\mathrm{b}}$ & 0.825 & 0.385 & 0.859 & $\mathbf{0 . 2 7 8}$ \\
$\mathbf{x}_{01} \rightarrow \mathbf{x}_{1}$ & $(1-\lambda) w_{\mathrm{p}}+\lambda w_{\mathrm{b}}$ & 0.95 & 0.741 & 0.088 & $\mathbf{0 . 0 6 0}$ \\
& $(1-\lambda) w_{\mathrm{c}}+\lambda w_{\mathrm{p}}$ & 0.855 & 0.216 & $\mathbf{0 . 1 8 1}$ & 0.495 \\
\hline$(\mathbf{b})$ & $(1-\lambda) w_{\mathrm{c}}+\lambda w_{\mathrm{b}}$ & 0.85 & $\mathbf{0 . 0 1 0}$ & 0.828 & 0.132 \\
$\mathbf{x}_{02} \rightarrow \mathbf{x}_{2}$ & $(1-\lambda) w_{\mathrm{p}}+\lambda w_{\mathrm{b}}$ & 0.75 & 0.220 & $\mathbf{0 . 0 9 8}$ & 0.106 \\
& $(1-\lambda) w_{\mathrm{c}}+\lambda w_{\mathrm{p}}$ & 0.617 & $\mathbf{0 . 0 0 1}$ & 0.002 & 0.261 \\
\hline
\end{tabular}

b) Three-objective Problems: The three proposed metrics can be aggregated together as follows:

$$
w_{\lambda}=\left(1-\lambda_{2}\right)\left(1-\lambda_{1}\right) w_{\mathrm{c}}+\lambda_{2}\left(1-\lambda_{1}\right) w_{\mathrm{p}}+\lambda_{1} w_{\mathrm{b}}
$$

with $\lambda_{1}$ and $\lambda_{2} \in[0 ; 1]$. When considering more than two objectives, the non-dominated solutions is the convex hull of the Pareto optimal surface that is explored as the weight set $\left\{\lambda_{1}, \lambda_{2}\right\}$ is varied. Actually, with more than two objectives the Pareto frontier is more difficult to handle and to illustrate easily. The compromise solution is located at $\widehat{\lambda}_{1}=0.52$ and $\widehat{\lambda}_{2}=0.95$, and provides the following normalized geodesic length: $L_{w_{\mathrm{c}}}=0.362, L_{w_{\mathrm{p}}}=0.106$ and $L_{w_{\mathrm{b}}}=0.288$. The corresponding navigation path is then the one that minimizes the geodesic distance to the utopian point, and thus allows to best fit the three objectives.

3) Discussions: From the multi-objective optimization problems considering the case Fig.9(a), $\mathbf{x}_{01} \rightarrow \mathbf{x}_{1}$, it appears two distinct interpretations. First, the compromise weight $\widehat{\lambda}_{2}$ is close to 1 . It supposes that the centerline metric $w_{\mathrm{c}}$ does not have a great influence in the three-objective problem given in (23). This interpretation appears to be consistent wrt. results from bi-objective problems, where the compromise weights $\widehat{\lambda}$ are also close to 1 . From the compromise weights analysis it appears that the centerline objective has less significance wrt. the other metrics. Actually, results from single objective show that globally each geodesic remains not far from the centerline pathway. Furthermore, this interpretation shows that the belief objective, $w_{\mathrm{b}}$, is the fully satisfied. Secondly, from the investigation of the normalized geodesic length at the compromise weight, $\widehat{\lambda}$, location, it appears that the power cost function $w_{\mathrm{p}}$ seems satisfied, as it has a low geodesic length, $L_{w_{\mathrm{p}}}=0.106$. In contrast, centerline objective has the largest length, $L_{w_{c}}=0.362$, which could be interpreted by a lower satisfaction than the other objectives at the compromise solution. In addition, objectives of power and centerline appear close to each other, since their compromise solution is close to the utopia. A similar analysis between objectives of power and belief allows us to consider that power objective is the less relevant for the case (1), $\mathbf{x}_{01} \rightarrow \mathbf{x}_{1}$, shown in Fig.11. Actually, in the considered multi-objective cases, the magnetic microrobot navigates in a steady flow, and thereby the drag force does not induce a significant constraint, particularly against the uncertainty constraint. In such context, the main objectives are to satisfy first the metrics of belief, and then the centerline goals where the $\mathbf{f}_{\mathrm{Ext}}$ can be neglected [15]. The power metric has a more important impact when the fluid 
flow implies significant forces on the microrobot dynamics (see Fig.9b).

The presence of distinctive solutions while the weight is varying (such as in the case (2) $\mathbf{x}_{02} \rightarrow \mathbf{x}_{2}$ depicted in Fig.11) is related to a non-convexity in the Pareto frontier. It is then more difficult to fully handle an ideal solution. Especially, the well-known drawbacks of the weighted sum method (18), are i) that the optimal solution distribution is not uniform; and ii) that optimal solutions in non-convex regions are not detected. More advanced multi-objective optimization techniques have to be used [22].

\section{Magnetic Resonance Navigation ExPeriments}

The proposed MRN framework has been experimentally

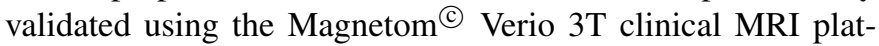
form (see Fig. 4). The considered MRN is defined as a motion around the vascular phantom. A spherical microrobot of radius $r=750 \mu \mathrm{m}$ has been introduced into the phantom setup and placed in the focus of the MRI bore. Propulsion and sensing MRI sequences are similar to the paragraph IV-C. As mentioned, uncertainties are here the most significant constraints. Therefore, the MRN planning is reduced to the bi-objective problem (22) considering the centerline $w_{x}=w_{\mathrm{c}}$ and belief $w_{y}=w_{\mathrm{b}}$ metrics. Moreover, a compromise weight of $\widehat{\lambda}=0.825$ is used to compute automatically the navigation path, as it is the one that best meets the two objectives. To generate a complex pathway, several waypoints are defined in the vascular-like environment. Then, the magnetic microrobot has to follow the predefined reference path using a simple proportional-integral (PI) controller. To guide the microrobot to follow the reference path the magnetic gradient depicted in Fig. 13 is generated with the coils of the MRI scanner. For the sake of clarity only the propulsion gradient sequences are reported here. As shown in Fig. 14 and in the supplementary video, the MRN system is able to steer efficiently the microrobot along the reference path. First, from its initial position $\left(\mathbf{x}_{\text {init }}\right)$ the device has to converge to the reference path. Once the geodesic is reached, the microrobot remains globally close to it. These results, with very few user interaction, validate the proposed framework as a step towards autonomous MRN.

Moreover, let us recall that the beliefs metric $w_{\mathrm{b}}$ is a measurement of the uncertainties on the microrobot tracking. Hence, $w_{\mathrm{b}}$ helps to predict the covariance matrix $\Sigma_{\mathbf{y}}$ in the sensing process. The ellipses in Fig. 14 describe the prediction of the $\Sigma_{\mathbf{y}}$ along the measured position from the sensing modules. Red ellipse is related to high uncertainty, while blue ellipse to low uncertainty. Based on the dynamic model (7) (cf. Section II-B), a Kalman filter (KF) can be used to estimate the microrobot position. Particularly, in robotics, the KF is the most suited for tracking, localization, and navigation problems. Indeed, the KF works best i) with well-defined state descriptions, where observation and time-propagation models are well understood; and ii) where all errors are Gaussian distributions. Fig. 14 also illustrates the measured position and the KF corrected localization. Errors between the reference path and measurements, as well as wrt. the KF-tracked position are presented in Fig. 15. With the MR-tracked measured

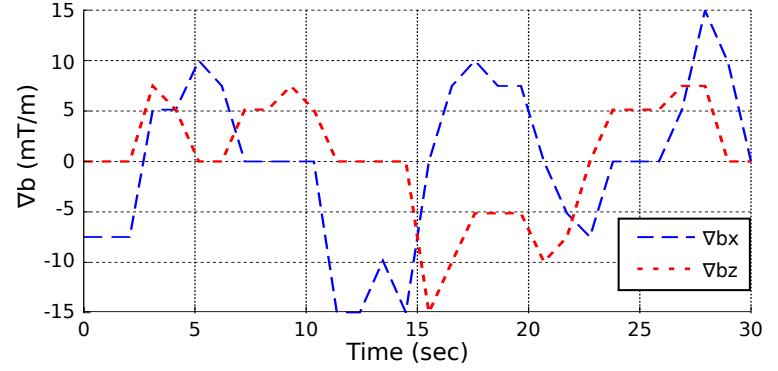

Fig. 13. Generated magnetic gradient to steer the magnetic microrobot along the reference path.

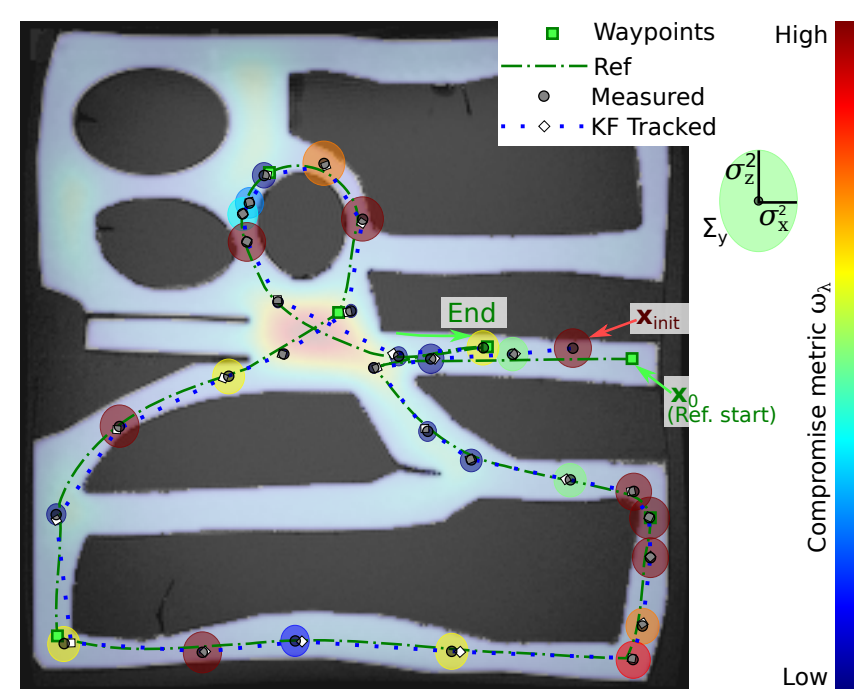

Fig. 14. 2D Magnetic Resonance Navigation along a reference path of a spherical magnetic microrobot of radius $r=750 \mu \mathrm{m}$. The image background shows the compromise metric: (blue) low metric value and (red) high value.

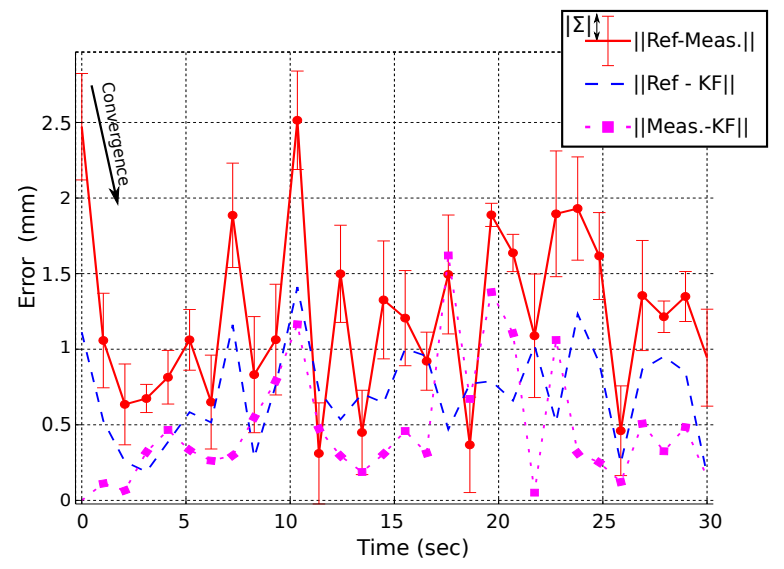

Fig. 15. Errors between the reference path and the measurements, and against the Kalman filtered localization.

value the average error is $1.249 \mathrm{~mm}$, whereas considering $\mathrm{KF}$-correction the mean error is $0.851 \mathrm{~mm}$. These results suggest that the KF correction provides a good estimate of the microrobot localization thanks to a suitable prediction of the microrobot's belief in its location. The microrobot tracking error appears greater than those considered in the sensing capability evaluation (cf. paragraph IV-B). Actually, 


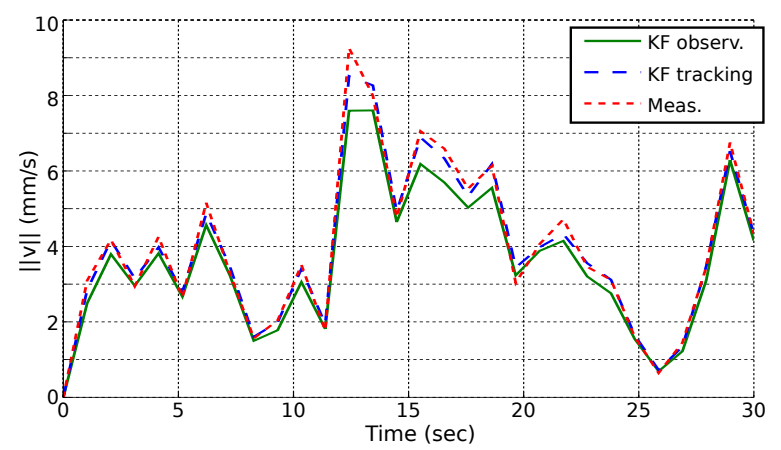

Fig. 16. The magnetic microrobot velocities.

the tracking algorithm is here designed upon a template stack of static artifacts. However, when the magnetic microrobot is moving, the artifact signature leaves a magnetic remnant of trail, as shown in Fig. 7c.

In addition, according to the system dynamic model (7) the KF allows the observation of the entire systems state $(\mathbf{v}, \mathbf{x})^{T}$ which includes the microrobot velocity v. Fig. 16 shows the motion of the microrobot computed from the measurements, the KF-corrected localization and the KF predicted velocity state. Once again, the use of the system's model together with an a priori knowledge of the uncertainty map improves substantively the MRN efficiency. Therefore, this could be a first step towards the design of an MRN-based medical SLAM (simultaneous localization and mapping) approach.

\section{Conclusions}

In this study, an optimal magnetic resonance navigation frameworks integrated within a clinical MRI scanner are presented. The basic sensing and actuation modules are first evaluated to exhibit the MRN system capabilities to track and magnetically steer through the magnetic field gradient generated from the coils of a conventional MRI. From preoperative MRI data and system models, comprising the sensing uncertainties and the system dynamics, a robust and optimal navigation path is planned by addressing multi-objective problem. A deep analysis of the different objective functions and of the multi-objective navigation planning problem is presented. Especially, the weight set could be automatically selected by considering the compromise solution. The reliable pathway is then used to efficiently guide a magnetic microrobot to a targeted location. The overall MRN architecture functionalities has been experimentally verified using an integrated clinical MRI system. These experimental realization demonstrate that it is effectively possible to autonomously guide a magnetic microrobot using a conventional MRI scanner with only a software upgrade. Further investigation will address 3D and in vivo experimental validation.

\section{ACKNOWLEDGMENTS}

The authors would like to acknowledge the Dr A. Kluge (Pius Hospital, Oldunburg, Germany) and Prof. S. Fatikov (Division Microrobotics and Control Engineering, University of Oldenburg, Germany) for their supports on the use of the clinical MRI data. This work was supported by European Unions 7th Framework Program and its research area ICT2007.3.6 Micro/nanosystems under the project NANOMA (Nano-Actuactors and Nano-Sensors for Medical Applications).

\section{REFERENCES}

[1] P. Dario, M. C. Carrozza, A. Benvenuto, and A. Menciassi, "Microsystems in biomedical applications," J. of Micromechanics and Microengineering, vol. 10, no. 2, p. 235, 2000.

[2] B. J. Nelson, I. K. Kaliakatsos, and J. J. Abbott, "Microrobots for minimally invasive medicine," Annual rev. of biomed. eng., vol. 12, pp. 55-85, 2010.

[3] M. P. Kummer, J. J. Abbott, B. E. Kratochvil, R. Borer, A. Sengul, and B. J. Nelson, "Octomag: An electromagnetic system for 5-dof wireless micromanipulation,” IEEE Trans. Robot., vol. 26, no. 6, pp. 1006-1017, 2010.

[4] Z. Zhang, F. Long, and C.-H. Menq, "Three-dimensional visual servo control of a magnetically propelled microscopic bead," IEEE Trans. Robot., vol. 29, no. 2, pp. 373-382, 2013.

[5] S. Martel, J.-B. Mathieu, O. Felfoul, A. Chanu, E. Aboussouan, S. Tamaz, P. Pouponneau, L. Yahia, G. Beaudoin, G. Soulez et al., "Automatic navigation of an untethered device in the artery of a living animal using a conventional clinical magnetic resonance imaging system," Appl. Phy. Lett., vol. 90, no. 11, p. 114105, 2007.

[6] S. Tamaz, A. Chanu, J.-B. Mathieu, R. Gourdeau, and S. Martel, "Real-time MRI-based control of a ferromagnetic core for endovascular navigation," IEEE Trans. Biomed. Eng., vol. 55, no. 7, pp. 1854-1863, Jul. 2008 .

[7] K. Belharet, D. Folio, and A. Ferreira, Real-time software platform for in vivo navigation of magnetic micro-carriers using MRI system, ser. Biomaterials. Cambridge: Woodhead Publishing, Oct. 2012, no. 51, ch. 11.

[8] S. Martel, "Magnetic navigation control of microagents in the vascular network: Challenges and strategies for endovascular magnetic navigation control of microscale drug delivery carriers," IEEE Contr. Syst., vol. 33, no. 6, pp. 119-134, 2013.

[9] P. Pouponneau, G. Bringout, and S. Martel, "Therapeutic magnetic microcarriers guided by magnetic resonance navigation for enhanced liver chemoembilization: A design review," Annals of biomedical engineering, vol. 42, no. 5, pp. 929-939, 2014.

[10] A. Bigot, C. Tremblay, G. Soulez, and S. Martel, "Magnetic resonance navigation of a bead inside a three-bifurcation pmma phantom using an imaging gradient coil insert," IEEE Trans. Robot., vol. 30, no. 3, pp. 719-727, Jun. 2014.

[11] C. Dahmen, K. Belharet, D. Folio, A. Ferreira, and S. Fatikow, "MRIbased dynamic tracking of an untethered ferromagnetic microcapsule navigating in liquid," Int. J. of Optomechatronics, vol. 10, no. 2, pp. 73-96, 2016.

[12] B. A. Chronik, A. Alejski, and B. K. Rutt, "Design and fabrication of a three-axis edge ROU head and neck gradient coil," Magnetic resonance in medicine, vol. 44, no. 6, pp. 955-963, 2000.

[13] J. A. McNab, B. L. Edlow, T. Witzel, S. Y. Huang, H. Bhat, K. Heberlein, T. Feiweier, K. Liu, B. Keil, J. Cohen-Adad et al., "The human connectome project and beyond: Initial applications of $300 \mathrm{mt} / \mathrm{m}$ gradients," NeuroImage, vol. 80, pp. 234-245, 2013.

[14] J. A. Sethian, Level set methods and fast marching methods: evolving interfaces in computational geometry, fluid mechanics, computer vision, and materials science. Cambridge university press, 1999, vol. 3.

[15] K. Belharet, D. Folio, and A. Ferreira, "Simulation and planning of a magnetically actuated microrobot navigating in arteries," IEEE Trans. Biomed. Eng., vol. 60, no. 4, pp. 994-1001, Apr. 2013.

[16] L. Arcèse, M. Fruchard, and A. Ferreira, "Endovascular MagneticallyGuided Robots: Navigation Modeling and Optimization," IEEE Trans. Biomed. Eng., vol. 59, no. 4, pp. 977-987, 2012.

[17] K. Belharet, D. Folio, and A. Ferreira, "Three-dimensional controlled motion of a microrobot using magnetic gradients," Adv. Robotics, vol. 25, no. 8, pp. 1069-1083, 2011.

[18] L. Arcèse, M. Fruchard, and A. Ferreira, "Adaptive controller and observer for a magnetic microrobot," IEEE Trans. Robot., vol. 29, no. 4, pp. 1060-1067, Aug. 2013.

[19] H. Marino, C. Bergeles, and B. J. Nelson, "Robust electromagnetic control of microrobots under force and localization uncertainties," IEEE Trans. Autom. Sci. Eng., vol. 11, no. 1, pp. 310-316, 2014. 
[20] H. Takeda, C. Facchinetti, and J.-C. Latombe, "Planning the motions of a mobile robot in a sensory uncertainty field," IEEE Trans. Pattern Anal. Mach. Intell., vol. 16, no. 10, pp. 1002-1017, 1994.

[21] N. Roy and S. Thrun, "Coastal navigation with mobile robots," Adv. in Neural Processing Syst., vol. 12, no. 12, pp. 1043-1049, 1999.

[22] R. T. Marler and J. S. Arora, "Survey of multi-objective optimization methods for engineering," Structural and multidisciplinary optimization, vol. 26, no. 6, pp. 369-395, 2004. 